Supporting information

\title{
An 808 nm NIR Laser Excited Upconversion Nanoplatform for Combinatory Photodynamic and Chemotherapy with Deep Penetration and Acid Bursting Release Performance
}

Yingtao Zhong,,${ }^{\dagger} \ddagger$ Yun Dong,,${ }^{\dagger} \sharp$ Tie Chen, ${ }^{\dagger}$ Lingzhi Yang, ${ }^{\dagger}$ Min Yao, ${ }^{\dagger}$ Yunshi Zhi, ${ }^{\dagger}$ Haoyi Yang, ${ }^{\dagger}$ Jian Zhang, ${ }^{\dagger}$ and Wenchuan $\mathrm{Bi}^{*}, \dagger$

${ }^{\dagger}$ School of Pharmaceutical Sciences, Health Science Center, Shenzhen University, Shenzhen 518055, China

\section{Corresponding Author}

*Dr. Wenchuan Bi, School of Pharmaceutical Sciences, Health Science Center, Shenzhen University, Shenzhen 518055, China.

Email address: cathybcc1106@gmail.com

\section{Author Contributions}

These authors contributed equally. 
(a)

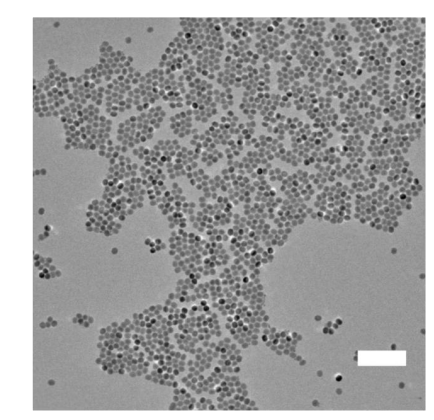

(d)

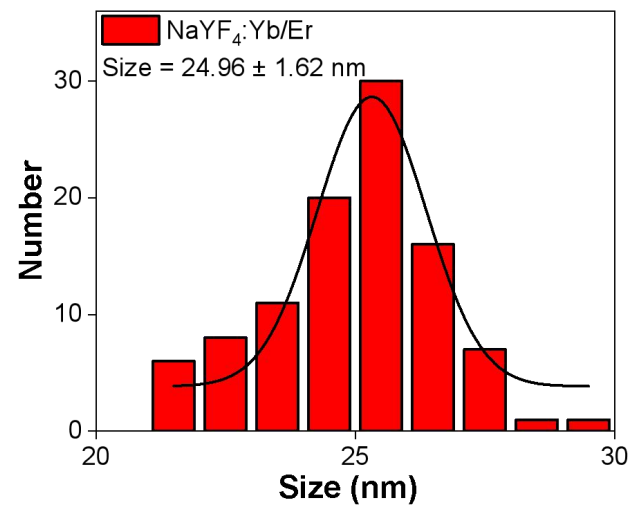

(c)

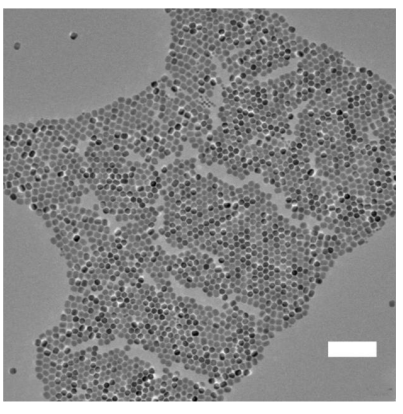

(e)

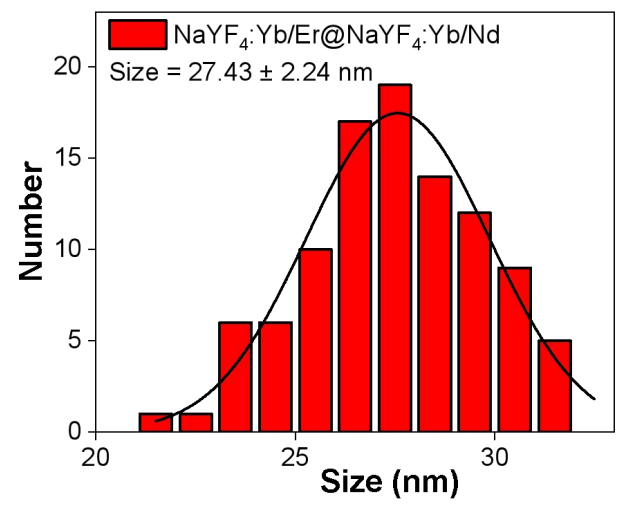

(f)

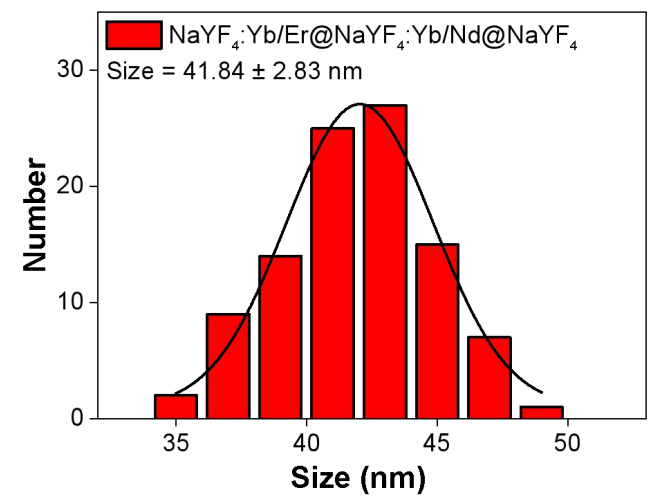

Figure S1. TEM images and particle size of (a) (d) $\mathrm{NaYF}_{4}: \mathrm{Yb} / \mathrm{Er}$, (b) (e) NaYF4:Yb/Er@NaYF4:Yb/Nd, and (c) (f) NaYF4:Yb/Er@ NaYF4:Yb/Nd@NaYF4. 
(a)

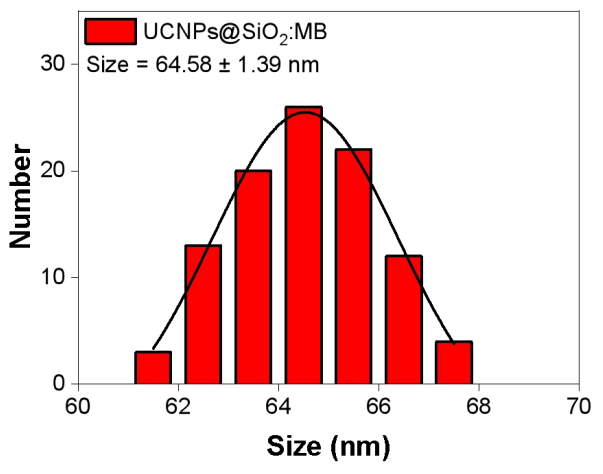

(b)

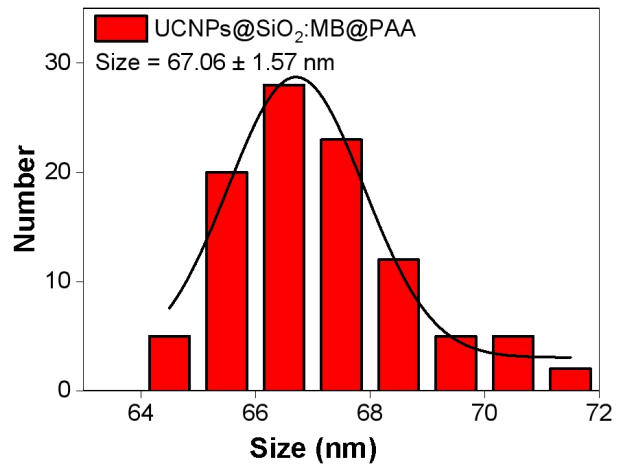

(c)

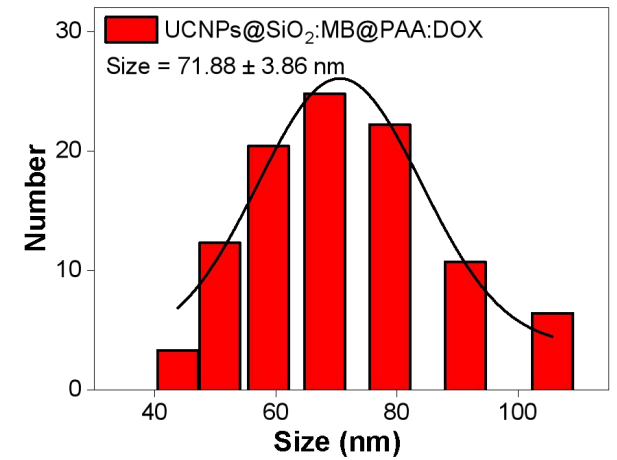

Figure S2. The statistics of particle size of (a) UCNPs@SiO $\mathrm{SiO}_{2}: \mathrm{MB}$, (b) UCNPs@SiO $2: \mathrm{MB} @ \mathrm{PAA}$ and (c) UCNPs@SiO $2: \mathrm{MB} @ \mathrm{PAA}: \mathrm{DOX}$, respectively.

S3. The calculation of FRET efficiency: The FRET efficiency can be calculated according to the equation:

$$
\text { FRET efficiency }(\%)=\frac{\mathrm{I}_{0}-\mathrm{I}_{1}}{\mathrm{I}_{0}} \times 100 \%
$$

$\mathrm{I}_{0}$ and $\mathrm{I}_{1}$ represent the integrated intensities of emission peak intensity at $650 \mathrm{~nm}$ of the nanoparticle before and after MB loading, respectively, and were calculated by origin version 2018. 
(a)

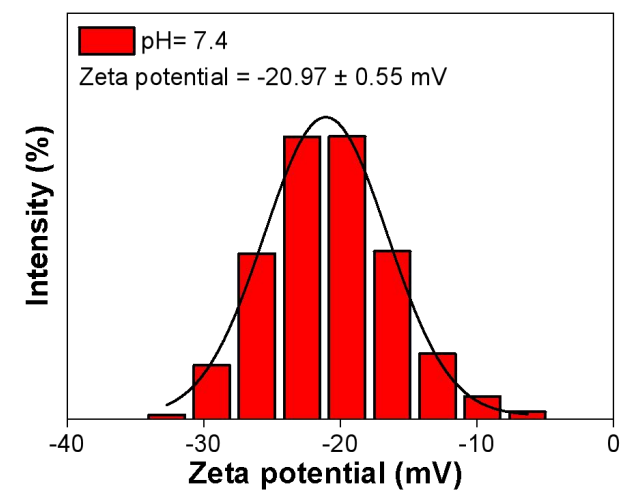

(b)

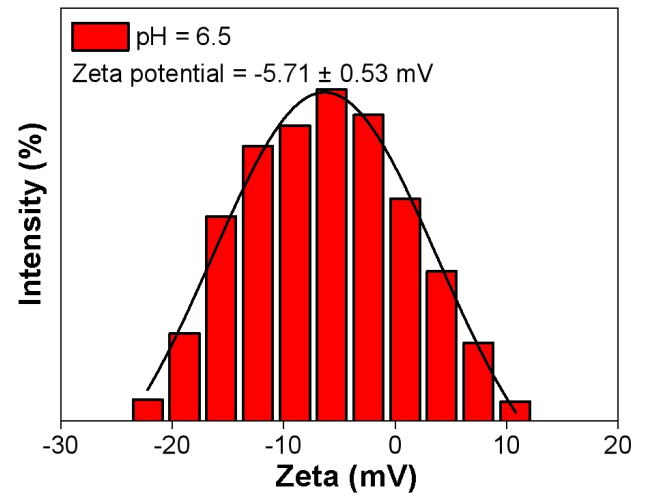

(c)

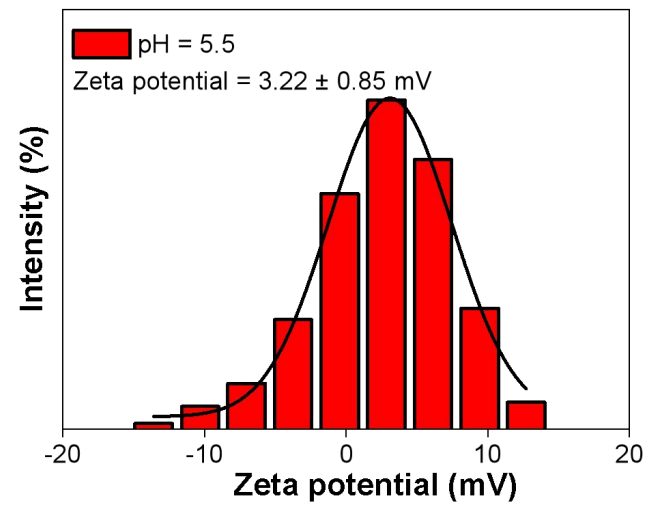

Figure S4. The zeta potential of (a) UCNPs@SiO $2: \mathrm{MB} @ \mathrm{PAA}$ at $\mathrm{pH}=7.4$, (b) $\mathrm{pH}=$ 6.5 , and (c) $\mathrm{pH}=5.5$. 
(a)
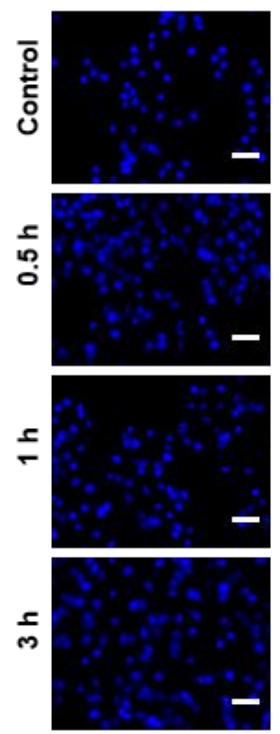
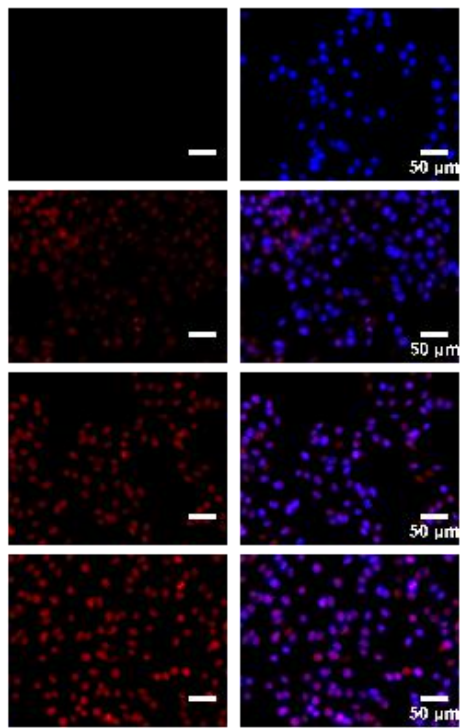

(b)
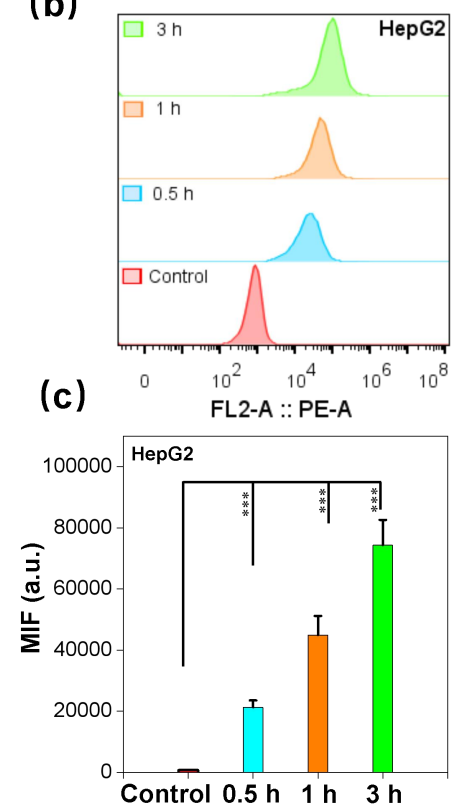

Figure S5. (a) Fluorescent images and (b) Flow cytometry histograms of HepG2 cells incubated with UCNPs@SiO :MB@PAA:DOX at different treatment time; (c) Mean fluorescence intensity at different treatment time. (Data are expressed as the mean \pm $\mathrm{SD}, * * * p<0.001)$ 

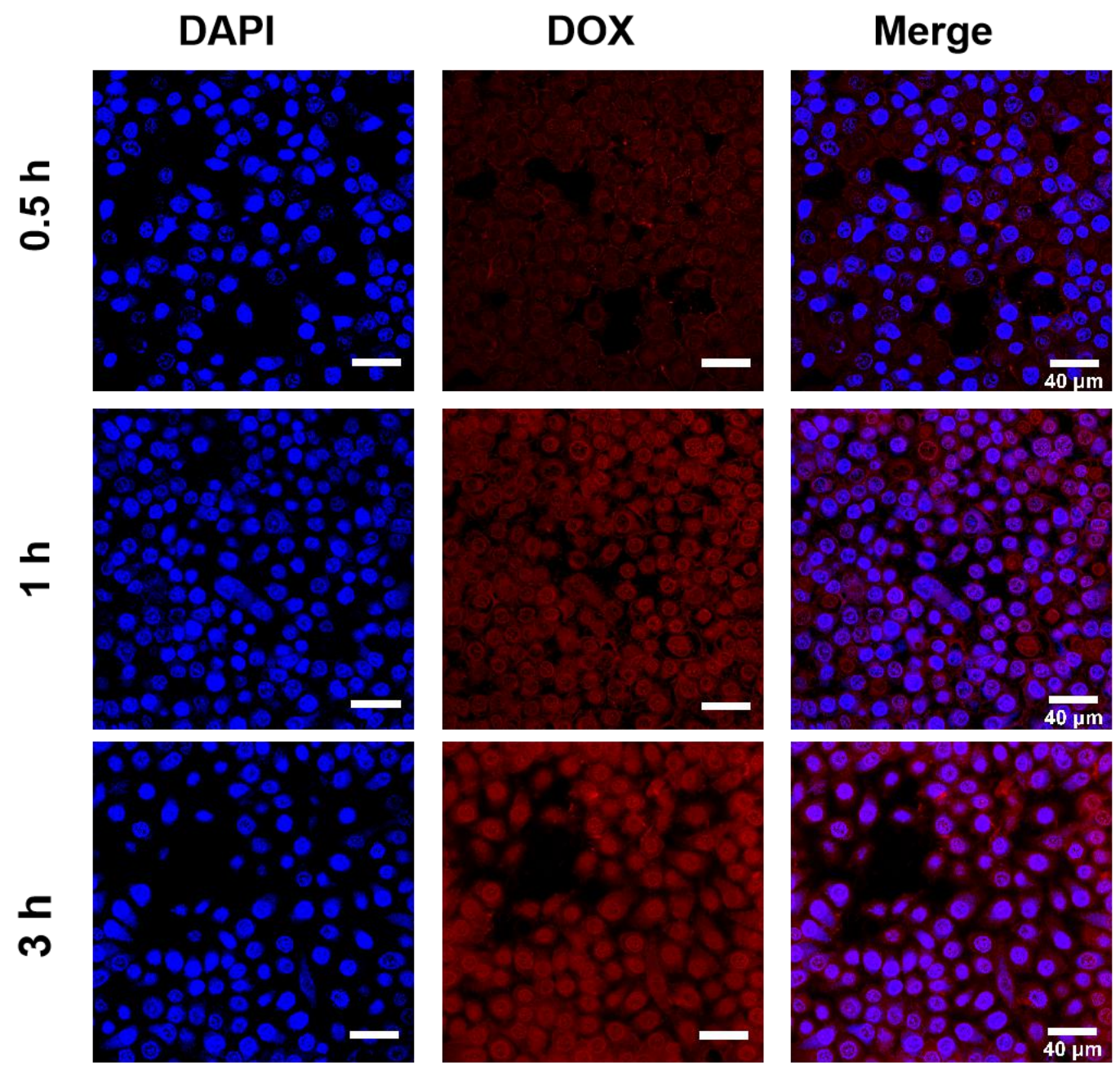

Figure S6. CLSM images of Hela cells incubated with UCNPs@SiOz:MB@PAA:DOX at different treatment time. 
(a)

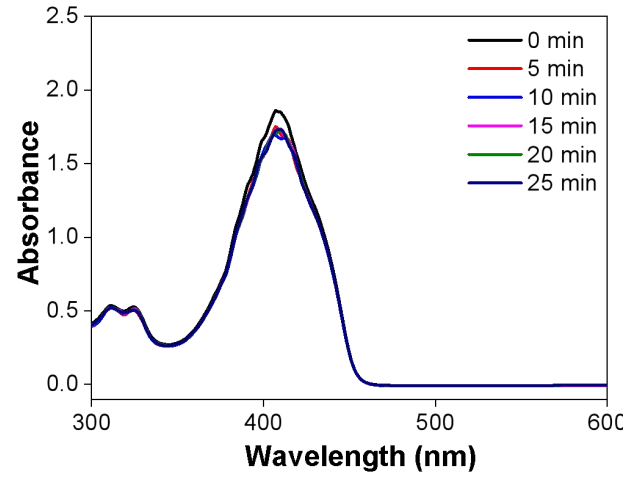

(b)

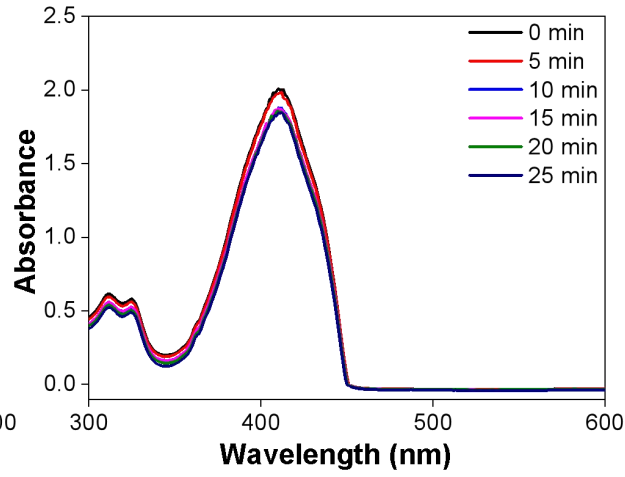

(c)
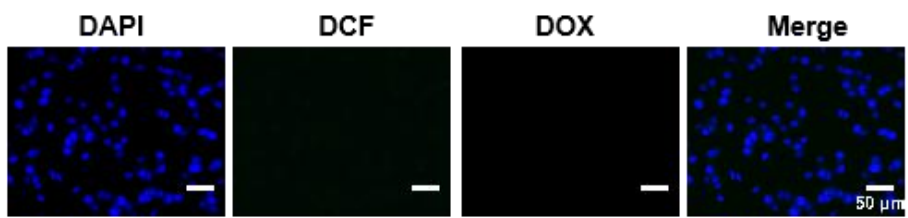

UCNPs@SiO
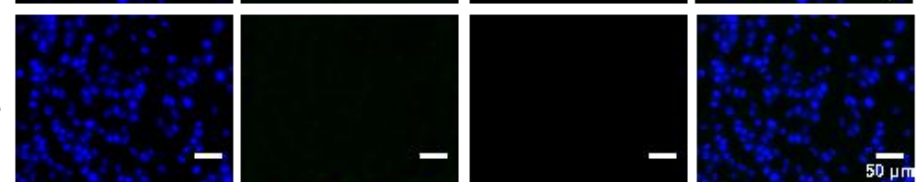
UCNPs@SiO
+Laser
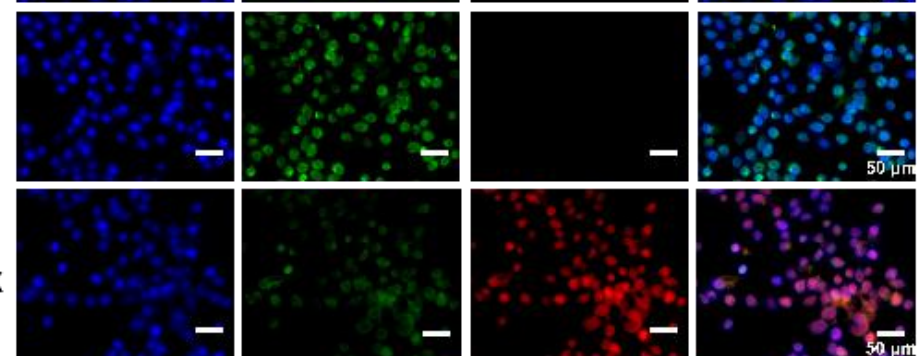

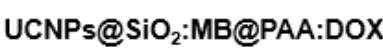
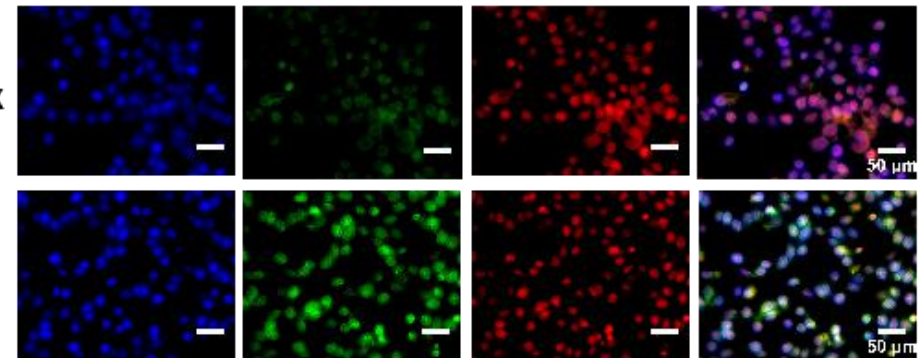

Figure S7. (a) Absorption spectra of DPBF incubated with UCNPs@SiO $\mathrm{Si}_{2} @ \mathrm{PAA}$ under laser irradiation for $25 \mathrm{~min}$ and (b) DPBF alone under irradiation at $808 \mathrm{~nm}$ NIR laser for $25 \mathrm{~min}$; (c) The ROS generation of HepG2 cells treated with UCNPs@SiO $2: \mathrm{MB} @ \mathrm{PAA}$ and UCNPs@SiO $: \mathrm{MB} @ \mathrm{PAA}: \mathrm{DOX}$ under light irradiation or in the dark. 
DAPI
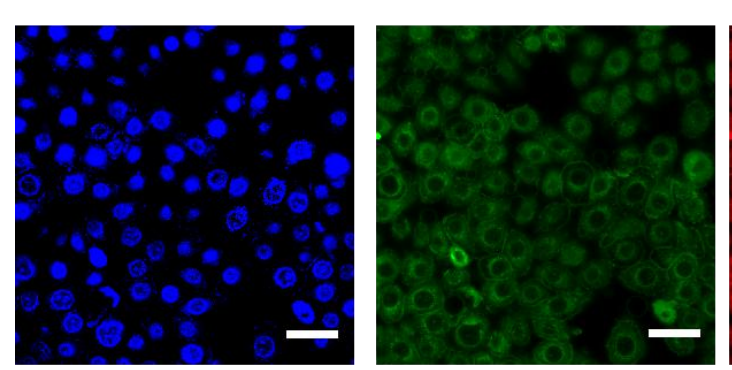

DOX

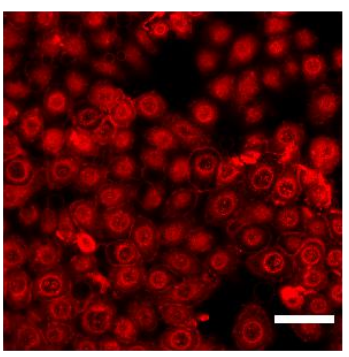

Merge

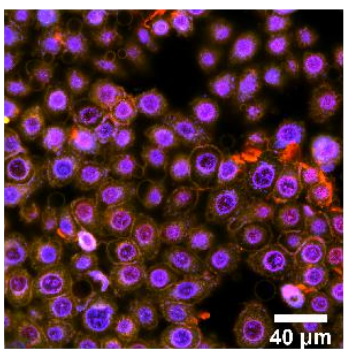

Figure S8. CLSM images of intracellular ROS production in Hela cells treated with UCNPs@SiO $2: M B @ P A A$ and UCNPs@SiO $:$ MB@PAA:DOX under light irradiation.

(a)

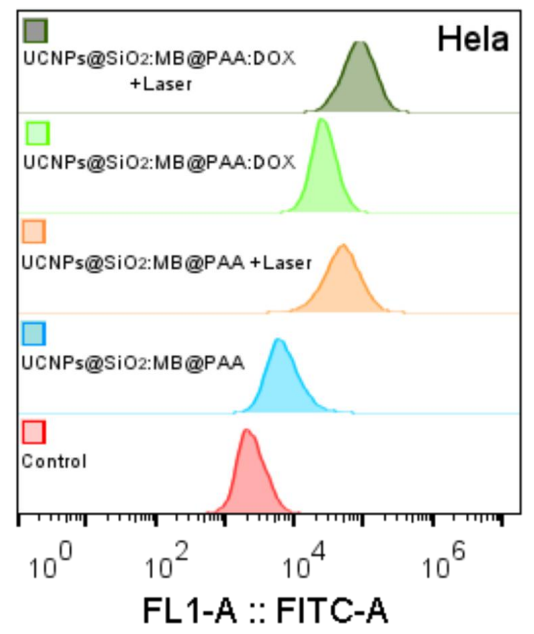

(b)

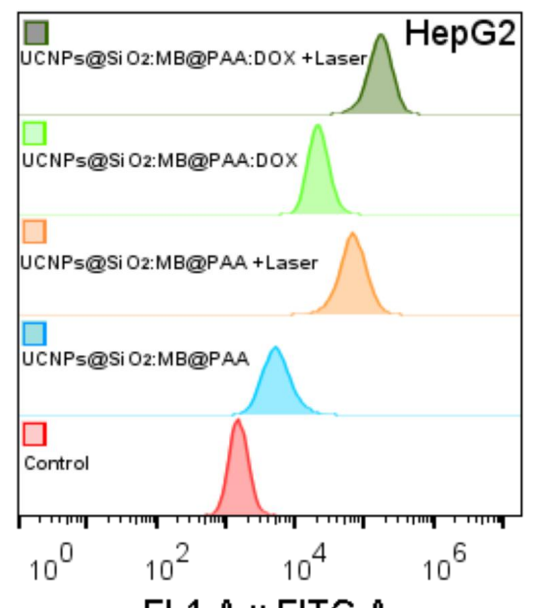

FL1-A :: FITC-A

(c)

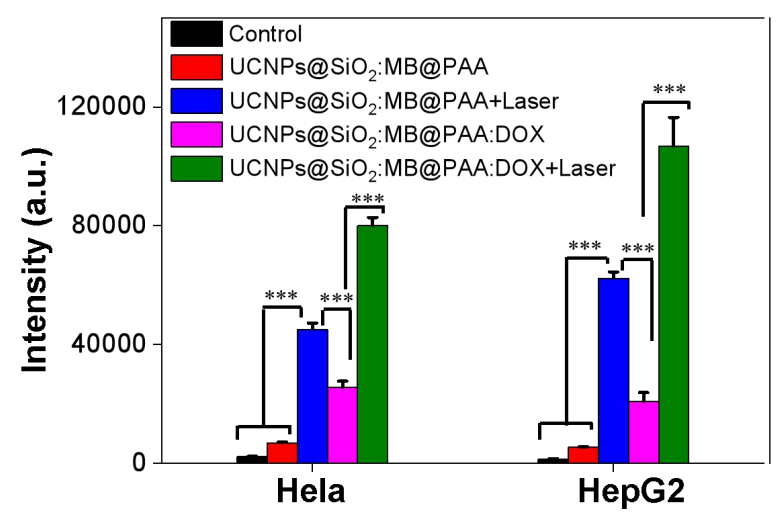

Figure S9. Flow cytometry histograms of ROS generation in (a) Hela and (b) HepG2 cells treated with different groups and (c) the statistics of mean fluorescence intensity for intracellular ROS generation. (Data are expressed as the mean $\pm \mathrm{SD}$, ${ }^{* * *} p<$ $0.001)$ 


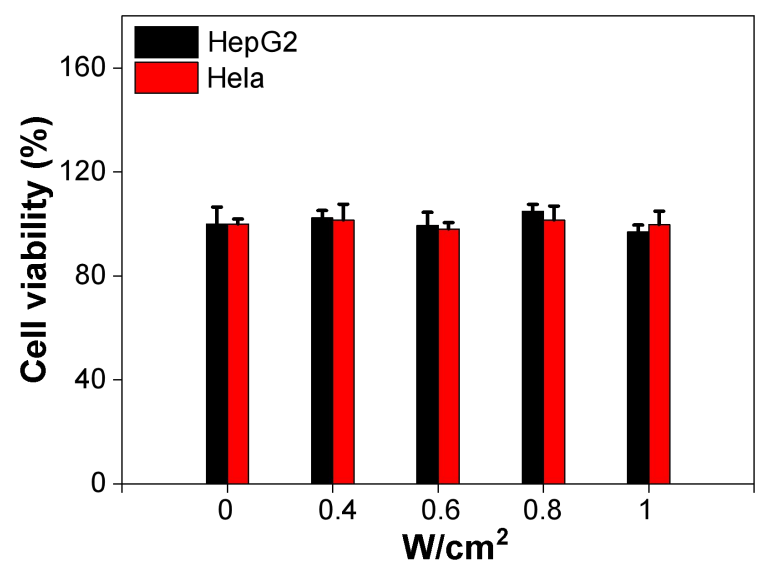

Figure S10. Cell viability of HeLa and HepG2 cells after $808 \mathrm{~nm}$ NIR laser irradiation at $0.4,0.6,0.8$ and $1 \mathrm{~W} \mathrm{~cm}^{-2}$ for $10 \mathrm{~min}$.

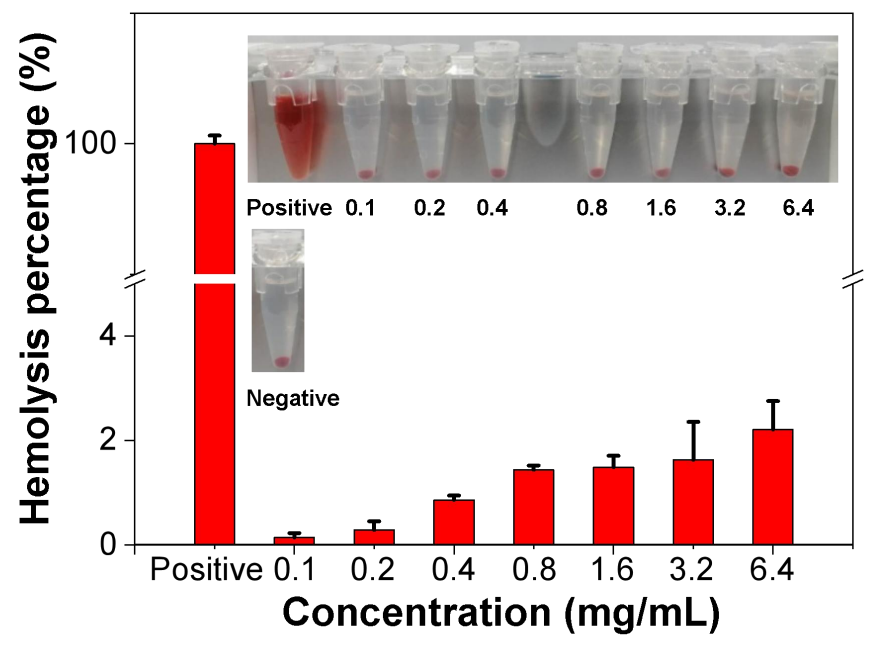

Figure S11. Hemolysis percentage of red blood cells after treated with UCNPs@SiO $2: \mathrm{MB} @$ PAA at different concentrations. 


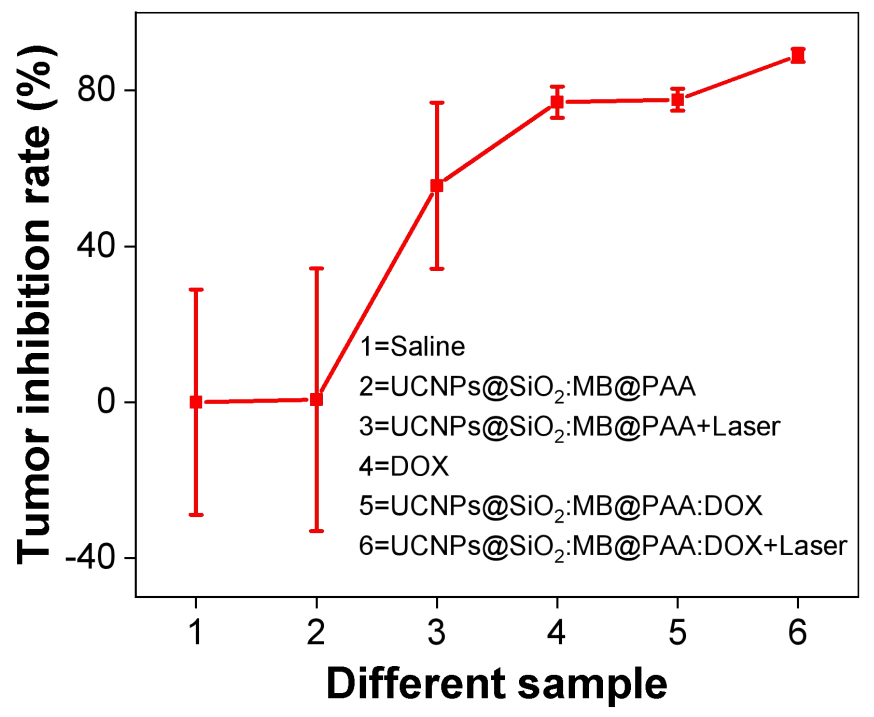

Figure S12. Tumor inhibition rate of different treatment groups. 


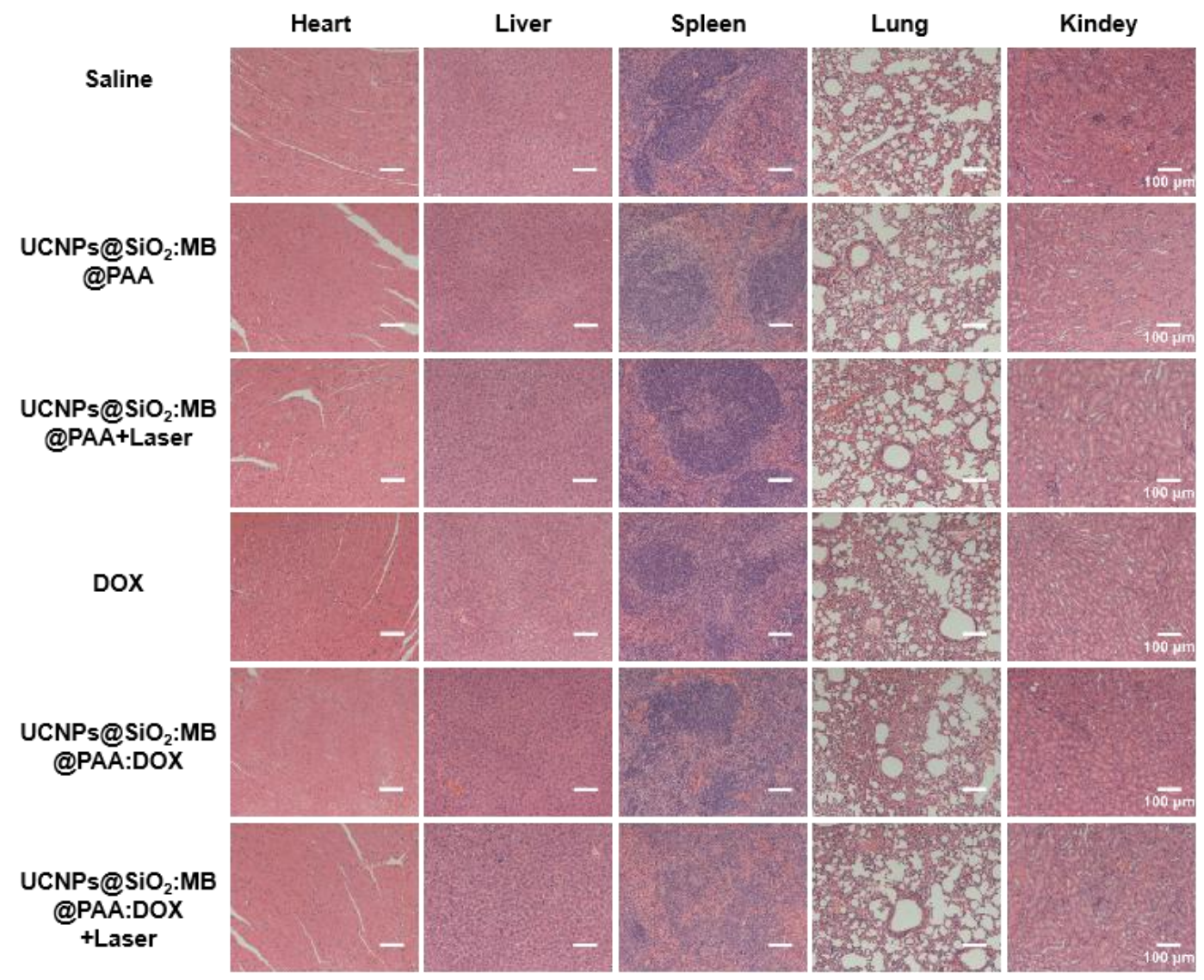

Figure S13. H\&E staining images of major organs after various treatments. 
(a)

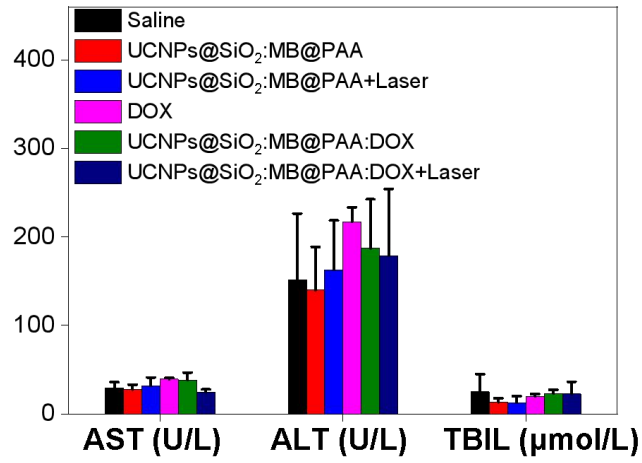

(b)

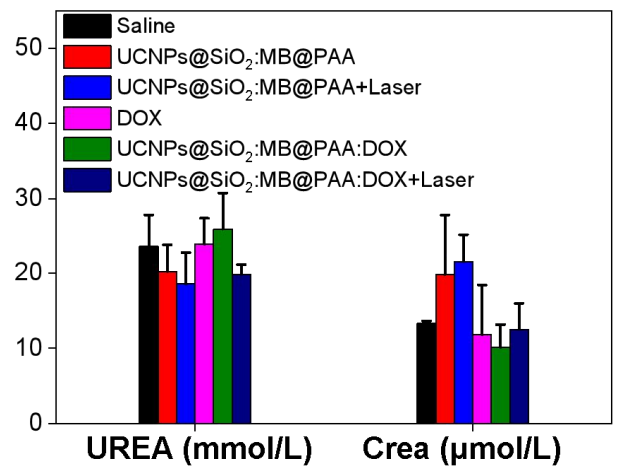

Figure S14. Biochemical analysis on the 14th day after the antitumor efficacy of different groups in vivo, (a) ALT, AST, TBIL for liver function, (b) UREA and CREA for renal function. 
Table S1. Summary of DOX IC S $_{50}$ Values in DOX-containing nanomedicines.

\begin{tabular}{|c|c|c|}
\hline Agents & Cell line & $\mathrm{IC}_{50}$ value $\left(\mu \mathrm{g} \mathrm{mL}^{-1}\right)$ \\
\hline C20/A80-DOX NP ${ }^{1}$ & Hela & $\sim 1.90$ \\
\hline C100B100-FA-DOX NP22 & Hela & $\sim 5.60$ \\
\hline Chitosan-hz-DOX ${ }_{0.070} \mathrm{NP}^{3}$ & Hela & $\sim 8.25$ \\
\hline FA-(C6 + Dox)-LP 4 & Hela & $\sim 6.21$ \\
\hline Tf-SL-DOX NP 5 & HepG2 & $\sim 11.83$ \\
\hline HA-Cyst-GA DOX-NP6 & HepG2 & $\sim 5.75$ \\
\hline $\mathrm{DOX}_{4} \mathrm{TNO}_{3} \mathrm{NP}^{7}$ & HepG2 & $\sim 2.65$ \\
\hline DOX-loaded CT9 NP ${ }^{8}$ & HepG2 & $\sim 2.50$ \\
\hline \multirow{2}{*}{ 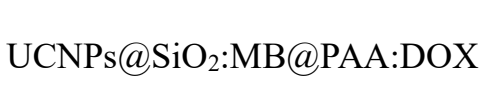 } & Hela & $\sim 1.56$ \\
\hline & HepG2 & $\sim 1.78$ \\
\hline
\end{tabular}

Table S2. Chemo-photodynamic Therapy to Achieve Half Maximal Inhibitory Concentration of tumor cell in Vitro.

\begin{tabular}{|c|c|c|c|}
\hline Agents & $\begin{array}{l}\text { Concentration } \\
\left(\mu \mathrm{g} \mathrm{mL}^{-1}\right)\end{array}$ & Cell line & Laser conditions \\
\hline Liposome $\mathrm{G}^{9}$ & $\sim 60.3$ & SKOV-3 & $\begin{array}{c}420 \mathrm{~nm}, 22.5 \mathrm{~mW} \mathrm{~cm}^{-2}, 30 \\
\min \end{array}$ \\
\hline $\mathrm{TCP} / \mathrm{PTX}^{10}$ & $\sim 12.7$ & U87-MG & $\begin{array}{c}\text { White light, } 0.1 \mathrm{~W} \mathrm{~cm}^{-2}, 10 \\
\min \end{array}$ \\
\hline Dox@BODIPY11 & $\sim 100$ & MDA-MB-231 & $665 \mathrm{~nm}, 20 \mathrm{~mW} \mathrm{~cm}{ }^{-2}, 20 \mathrm{~min}$ \\
\hline $\begin{array}{c}\text { MSN@SiNPs@TMPyP } \\
\text {-FA/Dox }{ }^{12}\end{array}$ & $\sim 60.73$ & MCF-7 & $655 \mathrm{~nm}, 300 \mathrm{~mW} \mathrm{~cm}{ }^{-2}, 90 \mathrm{~s}$ \\
\hline $\begin{array}{c}\text { Ce6/Dox@NPs-cRGD } \\
(\mathrm{CDNR})^{13}\end{array}$ & $\sim 86.8$ & MCF-7 & $670 \mathrm{~nm}, 0.25 \mathrm{~W} \mathrm{~cm}^{-2}, 5 \mathrm{~min}$ \\
\hline PS1b@DOX/Ce6 ${ }^{14}$ & $\sim 36.37$ & MCF-7 & $650 \mathrm{~nm}, 100 \mathrm{~mW} \mathrm{~cm}{ }^{-2}, 10 \mathrm{~min}$ \\
\hline RGD-NPs/Ce6 $6^{15}$ & $\sim 21.11$ & MCF-7 & $633 \mathrm{~nm}, 50 \mathrm{~mW} \mathrm{~cm}^{-2}, 5 \mathrm{~min}$ \\
\hline SMDSP$^{16}$ & $\begin{array}{l}\sim 39.15 \\
\sim 35.54\end{array}$ & $\begin{array}{l}\text { BEAS-2B } \\
\text { HepG2 }\end{array}$ & $808 \mathrm{~nm}, 1 \mathrm{~W} \mathrm{~cm}{ }^{-2}, 20 \mathrm{~min}$ \\
\hline Ce6/DOX@UCNPs ${ }^{17}$ & $\sim 43.33$ & A549 & $980 \mathrm{~nm}, 1 \mathrm{~W} \mathrm{~cm}^{-2}, 5 \mathrm{~min}$ \\
\hline
\end{tabular}


Table S3. Tumor Inhibition Rate of Chemo-Photodynamic Therapy in Vivo.

\begin{tabular}{|c|c|c|c|}
\hline Agents & Tumor-bearing mice & Inhibition rate $(\%)$ & Laser conditions \\
\hline R-SUZn NP + DOXil ${ }^{18}$ & PC-3 & 79.00 & $\begin{array}{c}980 \mathrm{~nm}, 34 \mathrm{~mW} \mathrm{~cm}^{-2}, \\
30 \mathrm{~min}\end{array}$ \\
\hline IT@MSN@RTM NP ${ }^{19}$ & $4 \mathrm{~T} 1$ & 64.00 & $\begin{array}{c}808 \mathrm{~nm}, 1.5 \mathrm{~W} \mathrm{~cm}^{-2} \\
5 \mathrm{~min}\end{array}$ \\
\hline DOX/F127-PheoA NP20 & B16 & 73.50 & $\begin{array}{c}670 \mathrm{~nm}, 200 \mathrm{~J} \mathrm{~cm}^{-2}, 5 \\
\min \end{array}$ \\
\hline $\begin{array}{c}\text { (UCNP@PFNS/AQ4N) } \\
@ \mathrm{MnCaP} \mathrm{NP}^{21}\end{array}$ & HepG2 & 83.00 & $\begin{array}{c}980 \mathrm{~nm}, 2.5 \mathrm{~W} \mathrm{~cm}^{-2} \\
1 \mathrm{~h}\end{array}$ \\
\hline $\begin{array}{c}\text { pPP-mCAuNCs@HA } \\
\text { NP }^{22}\end{array}$ & $4 \mathrm{~T} 1$ & 84.20 & $\begin{array}{c}670 \mathrm{~nm}, 270 \mathrm{~mW} \mathrm{~cm}{ }^{-2} \\
4 \mathrm{~min}\end{array}$ \\
\hline PTN NP 23 & BEL-7402 & 77.50 & $\begin{array}{c}660 \mathrm{~nm}, 50 \mathrm{~mW} \mathrm{~cm}^{-2}, \\
10 \mathrm{~min}\end{array}$ \\
\hline $\begin{array}{c}\mathrm{UCNPs} @ \mathrm{SiO}_{2}: \mathrm{MB} @ \mathrm{PAA} \\
\text { :DOX }\end{array}$ & Hela & 89.81 & $\begin{array}{c}808 \mathrm{~nm}, 1 \mathrm{~W} \mathrm{~cm}^{-2}, 20 \\
\text { min }\end{array}$ \\
\hline
\end{tabular}

\section{REFERENCE}

(1) Montero, N.; Perez, E.; Benito, M.; Teijon, C.; Teijon, J. M.; Olmo, R.; Blanco, M. D., Biocompatibility Studies of Intravenously Administered Ionic-Crosslinked Chitosan-BSA Nanoparticles as Vehicles for Antitumour Drugs. Int J Pharm 2019, 554, 337-351.

(2) Song, N.; Ding, M.; Pan, Z.; Li, J.; Zhou, L.; Tan, H.; Fu, Q., Construction of Targeting-Clickable and Tumor-Cleavable Polyurethane Nanomicelles for Multifunctional Intracellular Drug Delivery. Biomacromolecules 2013, 14, 4407-4419.

(3) Chen, C.; Zhou, J. L.; Han, X.; Song, F.; Wang, X. L.; Wang, Y. Z., A Prodrug Strategy Based on Chitosan for Efficient Intracellular Anticancer Drug Delivery. Nanotechnology 2014, 25, 255101.

(4) Sriraman, S. K.; Pan, J.; Sarisozen, C.; Luther, E.; Torchilin, V., Enhanced Cytotoxicity of Folic Acid-Targeted Liposomes Co-Loaded with C6 Ceramide and Doxorubicin: In Vitro Evaluation on HeLa, A2780-ADR, and H69-AR Cells. Mol Pharm 2016, 13, 428-437.

(5) Li, X.; Ding, L.; Xu, Y.; Wang, Y.; Ping, Q., Targeted Delivery of Doxorubicin Using Stealth Liposomes Modified with Transferrin. Int J Pharm 2009, 373, 116-123.

(6) Mezghrani, O.; Tang, Y.; Ke, X.; Chen, Y.; Hu, D.; Tu, J.; Zhao, L.; Bourkaib, N., Hepatocellular Carcinoma Dually-Targeted Nanoparticles for Reduction Triggered Intracellular Delivery of Doxorubicin. Int J Pharm 2015, 478, 553-568.

(7) Song, Q.; Tan, S.; Zhuang, X.; Guo, Y.; Zhao, Y.; Wu, T.; Ye, Q.; Si, L.; Zhang, Z., Nitric Oxide Releasing D-Alpha-Tocopheryl Polyethylene Glycol Succinate for Enhancing Antitumor Activity of Doxorubicin. Mol Pharm 2014, 11, 4118-4129. 
(8) Guo, Y.; Chu, M.; Tan, S.; Zhao, S.; Liu, H.; Otieno, B. O.; Yang, X.; Xu, C.; Zhang, Z., Chitosan-G-TPGS Nanoparticles for Anticancer Drug Delivery and Overcoming Multidrug Resistance. Mol Pharm 2014, 11, 59-70.

(9) Wang, Y.; Tian, N.; Li, C.; Hou, Y.; Wang, X.; Zhou, Q. Incorporation of 7-Dehydrocholesterol into Liposomes as a Simple, Universal and Efficient Way to Enhance Anticancer Activity by Combining Pdt and Photoactivated Chemotherapy. Chem Commun 2019, 55, 14081-14084.

(10) Yuan, Y.; Liu, B. Self-Assembled Nanoparticles Based on Pegylated Conjugated Polyelectrolyte and Drug Molecules for Image-Guided Drug Delivery and Photodynamic Therapy. ACS Appl Mater Interfaces 2014, 6, 14903-14910.

(11) Wang, S.; Li, J.; Ye, Z.; Li, J.; Wang, A.; Hu, J.; Bai, S.; Yin, J. Self-Assembly of Photosensitive and Chemotherapeutic Drugs for Combined Photodynamic-Chemo Cancer Therapy with Real-Time Tracing Property. Colloids and Surfaces A: Physicochemical and Engineering Aspects 2019, 574, 44-51.

(12) Li, S.; Zhang, Y.; He, X. W.; Li, W. Y.; Zhang, Y. K. Multifunctional Mesoporous Silica Nanoplatform Based on Silicon Nanoparticles for Targeted Two-Photon-Excited Fluorescence Imaging-Guided Chemo/Photodynamic Synergetic Therapy in Vitro. Talanta 2020, 209, 120552.

(13) He, Z.; Jiang, H.; Zhang, X.; Zhang, H.; Cui, Z.; Sun, L.; Li, H.; Qian, J.; Ma, J.; Huang, J. Nano-Delivery Vehicle Based on Chlorin E6, Photodynamic Therapy, Doxorubicin Chemotherapy Provides Targeted Treatment of HER-2 Negative, avß33-Positive Breast Cancer. Pharmacol Res 2020, 160, 105184.

(14) Yu, L.; Yang, Y.; Du, F. S.; Li, Z. C. ROS-Responsive Chalcogen-Containing Polycarbonates for Photodynamic Therapy. Biomacromolecules 2018, 19, 2182-2193.

(15) Zhang, Y.; Huang, F.; Ren, C.; Yang, L.; Liu, J.; Cheng, Z.; Chu, L.; Liu, J. Targeted Chemo-Photodynamic Combination Platform Based on the DOX Prodrug Nanoparticles for Enhanced Cancer Therapy. ACS Appl Mater Interfaces 2017, 9, 13016-13028.

(16) Sang, D.; Li, X.; Xu, Z.; Lin, H.; Guo, C.; Qu, F. Disrupted Intracellular Redox Balance with Enhanced ROS Generation and Sensitive Drug Release for Cancer Therapy. Biomater Sci 2020, 8, 6045-6055.

(17) Tian, G.; Ren, W.; Yan, L.; Jian, S.; Gu, Z.; Zhou, L.; Jin, S.; Yin, W.; Li, S.; Zhao, Y. Red-Emitting Upconverting Nanoparticles for Photodynamic Therapy in Cancer Cells under Near-Infrared Excitation. Small 2013, 9, 1929-1938.

(18) Gao, W.; Wang, Z.; Lv, L.; Yin, D.; Chen, D.; Han, Z.; Ma, Y.; Zhang, M.; Yang, M.; Gu, Y. Photodynamic Therapy Induced Enhancement of Tumor Vasculature Permeability Using an Upconversion Nanoconstruct for Improved Intratumoral Nanoparticle Delivery in Deep Tissues. Theranostics 2016, 6, 1131-1144.

(19) Wang, H.; Zhang, C.; Zhang, Y.; Tian, R.; Cheng, G.; Pan, H.; Cui, M.; Chang, J. An Efficient Delivery of Photosensitizers and Hypoxic Prodrugs for a Tumor Combination Therapy by Membrane Camouflage Nanoparticles. J Mater Chem B 2020, 8, 2876-2886.

(20) Zhang, C.; Zhang, J.; Qin, Y.; Song, H.; Huang, P.; Wang, W.; Wang, C.; Li, C.; Wang, Y.; Kong, D. Co-Delivery of Doxorubicin and Pheophorbide A by Pluronic 
F127 Micelles for Chemo-Photodynamic Combination Therapy of Melanoma. $J$ Mater Chem B 2018, 6, 3305-3314.

(21) Ji, Y.; Lu, F.; Hu, W.; Zhao, H.; Tang, Y.; Li, B.; Hu, X.; Li, X.; Lu, X.; Fan, Q.; Huang, W. Tandem Activated Photodynamic and Chemotherapy: Using $\mathrm{pH}$-Sensitive Nanosystems to Realize Different Tumour Distributions of Photosensitizer/Prodrug for Amplified Combination Therapy. Biomaterials 2019, 219, 119393.

(22) Yu, W.; He, X.; Yang, Z.; Yang, X.; Xiao, W.; Liu, R.; Xie, R.; Qin, L.; Gao, H. Sequentially Responsive Biomimetic Nanoparticles with Optimal Size in Combination with Checkpoint Blockade for Cascade Synergetic Treatment of Breast Cancer and Lung Metastasis. Biomaterials 2019, 217, 119309.

(23) Ji, C.; Gao, Q.; Dong, X.; Yin, W.; Gu, Z.; Gan, Z.; Zhao, Y.; Yin, M. A Size-Reducible Nanodrug with an Aggregation-Enhanced Photodynamic Effect for Deep Chemo-Photodynamic Therapy. Angew Chem Int Ed Engl 2018, 57, 11384-11388. 